\title{
Laryngeal mask ventilation in patient with Treacher Collins syndrome
}

\author{
Tsvetomir Marinov, MD, PhD \\ Department of Anesthesiology and Intensive Care \\ University Hospital "Queen Giovanna" - ISUL \\ Medical University - Sofia
}

\begin{abstract}
Introduction: Treacher Collins syndrome (TCS) is an autosomal dominant disorder of craniofacial development.

Case description: A 5-year-old female patient (weight $16.8 \mathrm{~kg}$, height $106 \mathrm{~cm}$ ), ASA III, was admitted to our hospital for plastic surgery of the ears. The preoperative evaluation revealed no significant personal or family history for adverse reaction to anesthetics. A general anesthesia was scheduled for this patient. Fentanyl $0.05 \mathrm{mg}$, propofol $50 \mathrm{mg}$ and succinylcholine $20 \mathrm{mg}$ were administered for anesthesia induction. After intubation $2.5 \%$ sevoflurane was used for anesthesia maintenance. Additional $0.025 \mathrm{mg}$ of fentanyl was administered for pain relief. The operation finished successfully within $55 \mathrm{~min}$. The vital signs had been stable during the operation. Ventilation through the operation was successfully performed through laryngeal mask classic № 2 .

Discussion: Management of patients with Treacher Collins syndrome is complicated and involves multiple disciplines working in concert to achieve a common outcome. When difficult intubation is anticipated, several alternatives to routine intubation are possible. In our study during maintenance of anesthesia, there was no evidence of instability of the LMA or increased contamination of the lower respiratory tract. During emergence from anesthesia, our results indicate that, compared with endotracheal anesthesia, airway management was easier using the LMA.

Conclusion: The laryngeal mask airway has become an important adjunct to airway management since its introduction. Ventilation through LMA can be safely used during operations in patients with Treacher Collins syndrome.
\end{abstract}

Key words: Treacher Collins syndrome, laryngeal mask, ventilation

\section{Introduction}

Treacher Collins syndrome (TCS) is an autosomal dominant disorder of craniofacial development. TCS occurs with an incidence of approximately $1 / 50000$ live births, however, more than 50\% of the cases are thought to arise as a result of a de novo mutation ${ }^{1}$. The clinical features of TCS are bilaterally symmetrical in nature and include: abnormalities of the external ears, atresia of external auditory canals, and malformation of the middle ear ossicles which result in bilateral conductive hearing loss 2; lateral downward sloping of the palpebral fissures, frequently with colobomas of the lower eyelids and a paucity of lid lashes medial to the defect; hypoplasia of the mandible and zygomatic complex; and cleft palate ${ }^{3}$.

On the basis that the tissues affected in TCS arise during early embryonic development from the first and second branchial arches, it has been proposed that the condition may arise from abnormal neural crest cell migration, improper cellular differentiation during development or an abnormality of the extracellular matrix ${ }^{4}$.

TCS can be inherited in an autosomal dominant or autosomal recessive manner. In autosomal dominant TCS about $55 \%-61 \%$ of cases have the disorder as the result of a de novo TCOF1, POLR1D, or POLR1B pathogenic variant. Each child of an individual with TCS has a $50 \%$ chance of inheriting the pathogenic variant. In autosomal recessive TCS the 
parents of a child with autosomal recessive TCS are obligate heterozygotes (i.e., carriers of one POLR1C or POLR1D pathogenic variant). At conception, each sib of an affected individual has a $25 \%$ chance of being affected, a $50 \%$ chance of being an asymptomatic carrier, and a $25 \%$ chance of being unaffected and not a carrier. Once the TCS-related pathogenic variant(s) have been identified in an affected family member, prenatal genetic testing are possible ${ }^{5}$.

Early operations focus on maintaining the airway, protecting the eyes, and supporting auditory neurological development. Later operations include staged reconstruction of the mouth, face, and external ear. Bimaxillary surgery can improve the maxillomandibular facial projection, but correction of malar, orbital rim, and temporal defects may be more difficult ${ }^{6}$.

\section{Case report}

Patients with Treacher Collins syndrome are considered difficult for intubation and airway instrumentation. It is reported in the case airway instrumentation through laryngeal mask during plastic operation of the ears in child.

A 5-year-old female patient (weight $16.8 \mathrm{~kg}$, height $106 \mathrm{~cm}$ ), ASA III, was admitted to our hospital for plastic surgery of the ears. The preoperative evaluation revealed no significant personal or family history for adverse reaction to anesthetics. A general anesthesia was scheduled for this patient. Fentanyl $0.05 \mathrm{mg}$, propofol $50 \mathrm{mg}$ and succinylcholine 20 $\mathrm{mg}$ were administered for anesthesia induction. After intubation $2.5 \%$ sevoflurane was used for anesthesia maintenance. Additional $0.025 \mathrm{mg}$ of fentanyl was administered for pain relief. The operation finished successfully within $55 \mathrm{~min}$. The vital signs had been stable during the operation.

Ventilation through the operation was successfully performed through laryngeal mask (LMA) classic № 2. The LMA was advanced by pressure against the hard palate to follow the oropharyngeal curve, advancing its tube with the other hand and oropharyngeal leak around LMA was detected by noise during manual bag ventilation. Mechanical ventilation was started using a ventilator (Drager Fabius Plus) in the volume control mode. A tidal volume of $120 \mathrm{ml}$, a respiratory rate of 20-22 and an inspiratory: expiratory ratio of 1:2 was used. Normocarbia was maintained (end tidal carbon dioxide $34-38 \mathrm{mmHg}$ ). Adequate ventilation was judged by end tidal carbon dioxide $\left(\mathrm{ETCO}_{2}\right)$ and expired tidal volume. Standard intraoperative monitoring was applied (non invasive blood pressure at 5 minutes interval, ECG, pulse oximetry and capnography). During maintenance of anesthesia, there was no evidence of instability of the LMA, compromising of ventilation or contamination of the lower respiratory tract (fig.1).

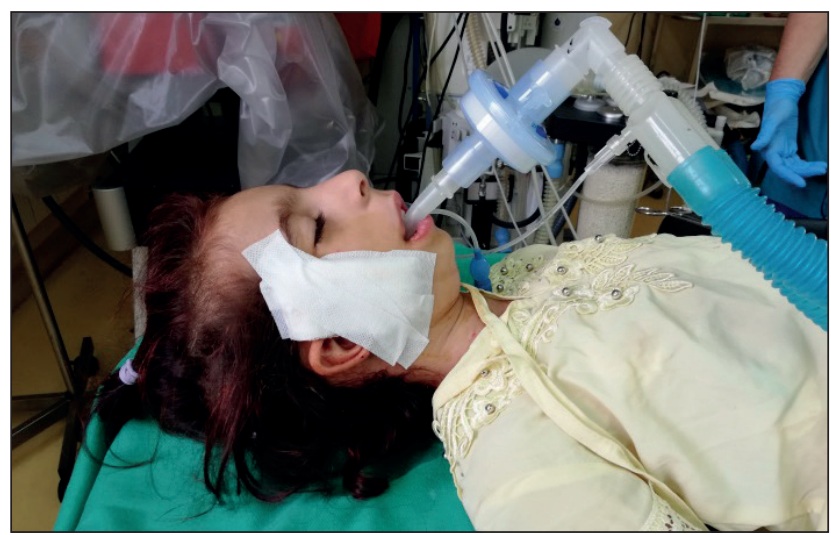

Fig.1. Ventilation through LMA in patient with Treacher Collins syndrome

Extubation was performed when the patient restores reflexes and spontaneous breathing with tidal volume of $5 \mathrm{ml} / \mathrm{kg}$. After extubation oxygen $4 \mathrm{~L} /$ min was administered via face mask. During the extubation there were no complications as laryngospasm, bronchospasm, coughing or desaturation. Discharge criteria from the operating room were normal respiratory pattern, adequate tidal volume and $\mathrm{SpO}_{2} \geq 98 \%$, then patient was transferred to post anesthetic care unit (fig.2).

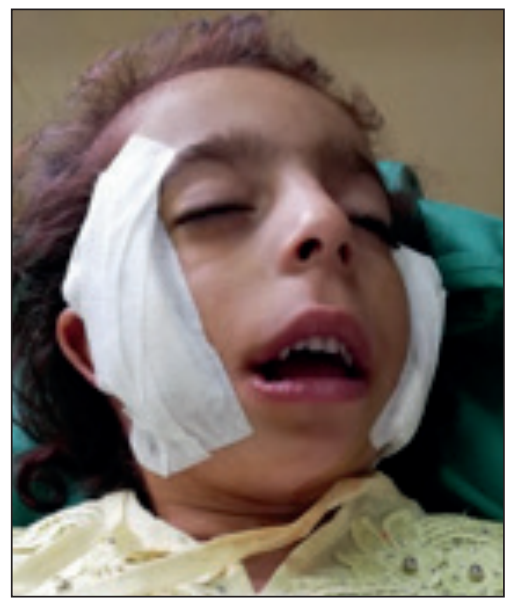

Fig.2. Patient with Treacher Collins syndrome after extubation

\section{Discussion}

Management of patients with Treacher Collins syndrome is complicated and involves multiple disciplines working in concert to achieve a common outcome. Bilateral conductive deafness is common 
in mandibulofacial dysostosis with or without atresia of the external auditory meatus. This deafness is due to a wide range of deformities of the ossicular chain associated with a characteristic reduction in the size of the middle ear cavity. The attic and antrum are particularly affected and usually have a slit-like appearance on coronal section tomograms 7. When difficult intubation is anticipated, several alternatives to routine intubation are possible. These include awake intubation with topical anesthesia, use of fiberoptic bronchoscopy, guided retrograde intubation, or tracheostomy. The LMA was designed by Brain in 1983 and has been in widespread use particularly in the UK as a new form of oral airway. The LMA can be inserted to the level of the larynx without laryngoscopy, and it permits positive pressure ventilation with a gas-tight seal up to $15 \mathrm{mmHg}$ of airway pressure ${ }^{8}$.

In our study during maintenance of anesthesia, there was no evidence of instability of the LMA or increased contamination of the lower respiratory tract. Postoperative respiratory complications are common in surgical patients. Trauma of airway maneuvers (laryngoscopy) and devices (tracheal tube) are among the different mechanisms involved in these incidences. These complications could be more serious in prolonged surgeries. A suitable airway control device must protect airways from aspiration. In elective surgeries, the risk of gastric content aspiration of LMA is not more than tracheal tube. In addition, these devices that have no access to the trachea do not traumatize the region. LMA as a supraglottic device in elective ENT operations is now considered as a standard choice. Although the duration of operation could be a lim- iting factor, the use of supraglottic device for elective prolonged ENT operations has been reported since $2001{ }^{9}$. The traditional fear of increased risk of gastric content aspiration with LMA does not exist anymore ${ }^{10}$. Advantages of the LMA are that there is no positive pressure cuff directly contacting the luminal surface of the trachea and the LMA does not require direct laryngoscopy for placement, in our study such anatomical advantages decreased the risk of dental and oral cavity injury during intubation and more rapid, better-controlled emergence from anesthesia.

The LMA is successfully applied when ventilation with a facial oxygen mask and endotracheal intubation fail. Successful use of the device has been reported in patients with a history of previous difficult intubation, in those with limited mouth opening, in patients with impaired mobility of the neck, in patients with Pierre-Robin syndrome, Treacher-Collins syndrome, and in those with juvenile arthritis. Treacher-Collins syndrome is characterized by choanal atresia. Choanal atresia is a rare abnormality presenting in the complete separation of the nasal cavity and the nasopharynx. This condition compromises facial oxygen mask ventilation, while LMA ventilation remains successful ${ }^{11,12}$.

\section{Conclusion}

The laryngeal mask airway has become an important adjunct to airway management since its introduction. Ventilation through LMA can be safely used during operations in patients with Treacher Collins syndrome.

\section{References}

1. Jones K.L., Smith D.W., Harvey M.A., Hall B.D., Quan L.. Older paternal age and fresh gene mutation: data on additional disorders, J. Pediatr., 1975, vol. 86 (pg. 84-88).

2. Phelps P.D., Poswillo D., Lloyd G.A.S.. The ear deformities in mandibulofacial dysostosis, Clin. Otolaryngol., 1981, vol. 6 (pg. 15-28).

3. Rovin S., Dachi S.F., Borenstein D.B., Cotter W.B.. Mandibulofacial dysostosis, a familial study of five generations, J. Pediatr., 1964 , vol. 65 (pg. 215-221)

4. Wiley M.J., Cauwenbergs P., Taylor I.M.. Effects of retinoic acid on the development of the facial skeleton in hamsters; early changes involving neural crest cells, Acta. Anat., 1983, vol. 116 (pg. 180-192).

5. Aljerian, Albaraa, and Mirko S. Gilardino. "Treacher collins syndrome.” Clinics in plastic surgery 46.2 (2019): 197-205.

6. Kobus, Kazimierz, and Piotr Wójcicki. "Surgical treatment of Treacher Collins syndrome." Annals of plastic surgery 56.5 (2006): 549-554.

7. PHELPS, PETER D., DAVID POSWILLO, and GLYN AS LLOYD. "The ear deformities in mandibulofacial dysostosis (Treacher Collins syndrome)." (1981): 15-28.

8. Ebata, Toshiya, et al. "Anaesthesia for Treacher Collins syndrome using a laryngeal mask airway." Canadian journal of anaesthesia 38.8 (1991): 1043-1045.

9. Maruyama K, Nakagawa H, Imanishi H, Kitamura A, Hayashida M. Comparison of postoperative pharyngeal morbidity using the Macintosh laryngoscope or AirWay Scope after mastectomy. J Anesth. 2011;25(5):773-6.

10. Nicholls M. ProSeal laryngeal mask airway use for prolonged middle ear surgery. Br J Anaesth. 2001;87(2):323-4.

11. Marinov, T., Belitova, M., Popov, T., Konov, D., \& Tsekova-Chernopolska, M. (2018). Laryngeal Mask or Endotracheal Intubation in Patients with Laryngeal Carcinoma. International Bulletin of Otorhinolaryngology, 14(1), 5-11.

12. Iliev, G., Milkov, M., Kerimov, K., Nedev, P., \& Ivanova, P. (2018). Rare case of choanal atresia in a 54-years-old female. International Bulletin of Otorhinolaryngology, 14(1), 42-44. 